\title{
Burst Mode FEL with the ETA-III Induction Linac*
}

\author{
C.J. Lasnier, S.L. Allen, B. Felker, M.E. Fenstermacher, S.W. Ferguson, S.D. Hulsey, E.B. Hooper, M.C. Jackson, M.A. \\ Makowski, W.H. Meyer, J.M. Moller, D.E. Petersen, S.E. Sampayan, B.W.Stallard, W.F. Fields \\ Lawrence Livermore National Laboratory, Livermore CA 94551 \\ K. Oasa \\ Japan Atomic Energy Research Institute, Tokai, Japan
}

\section{Abstract}

Pulses of $140 \mathrm{GHz}$ microwaves have been produced at a $2 \mathrm{kHz}$ rate using the ETA-III induction linac and IMP wiggler. The accelerator was run in bursts of up to 50 pulses at $6 \mathrm{MeV}$ and greatcr than $2 \mathrm{kA}$ peak current. A fecdback timing control system was used to synchronize acceleration voltage pulses with the electron beam, resulting in sufficient reduction of the corkscrew and energy sweep for efficient FEL operation. Peak microwave power for short bursts was in the range 0.5$1.1 \mathrm{GW}$, which is comparable to the single-pulse peak power of $0.75-2 \mathrm{GW}$ [1]. FEL bursts of more than 25 pulses were obtained.

\section{INTRODUCTION}

Pulses of high-power microwaves were generated using the ETA-III (an upgrade of ETA-II), a linear induction accelerator at the Lawrence Livermore National Laboratory, and the IMP (Intense Microwave Prototype) wiggler. The microwaves were produced as part of the MTX fusion energy project. To progress from single pulse operation to bursts of pulses at multi-kilohertz rates, modifications were made in the pulsedpower systems, and a new timing compensation scheme was implemented for synchronizing the accelcration voltage pulses with the electron beam.

The timing system was especially critical [4]. The electron beam provided most of the loading for the highvoltage pulse at the induction gaps. If the high voltage pulse did not arrive at the gap within $+/-10 \mathrm{nS}$ of the electron beam pulse, the unloaded voltage would have been too high, causing an arc and damaging insulators. To achieve the best acceleration of the beam, the high voltage pulse timing needed to be controlled to within $+/-2 \mathrm{nS}$ for each pulse of a burst. This was shown to be within the capability of the feedback timing compensation system. Note that it is not the standard deviation of timing measurements which is critical in preventing arc damage, it is the largest excursion of timing from the target value.

ETA-III was run in $2 \mathrm{kHz}$ bursts of up to 50 pulses. The electron beam reached peak currents of over $2 \mathrm{kA}$ at $6 \mathrm{MeV}$. An example of a long burst is shown in Fig 3. For FEL operation, the beam used in IMP wiggler with $7 \mathrm{~kW}$ of master oscillator power at $140 \mathrm{GHz}$ generated by a gyrotron.

\footnotetext{
${ }^{*}$ Work performed under the auspices of U.S.DOE by LLNL under contract W-7405-ENG-48
}

The FEL peak output power exceeded $1 \mathrm{GW}$ during many bursts. This is not as great as the highest power $(\approx 2 \mathrm{GW})$ measured during single-pulse operation [1]. Corkscrew and energy sweep of the beam during a burst remained within the acceptable range during short bursts, as evidenced by consistent FEL output (Fig 4). The experimental time to optimize operation was limited, so the longest recorded burst of microwaves contained slightly more than 25 pulses (Fig. 5).

\section{DESCRIPTION OF THE EXPERIMENT}

\section{A. The ETA-III Accelcrator}

The accelerator was configured in nearly the same way as for the previous 20-cell experiment $[1,2,3]$, except that 60 accelerator cells were in place to provide $6 \mathrm{MeV}$ beam energy. The high voltage pulses to drive the induction gaps were provided by four pulsed power systems, each consisting of a MAG-1D magnetic pulse compressor fed by a Pulsed Power Unit (PPU) (Fig 1). The PPU's were energized by DC power supplies typically run at $10-14 \mathrm{kV}$. A capacitor bank for each DC supply helped maintain voltage during bursts.

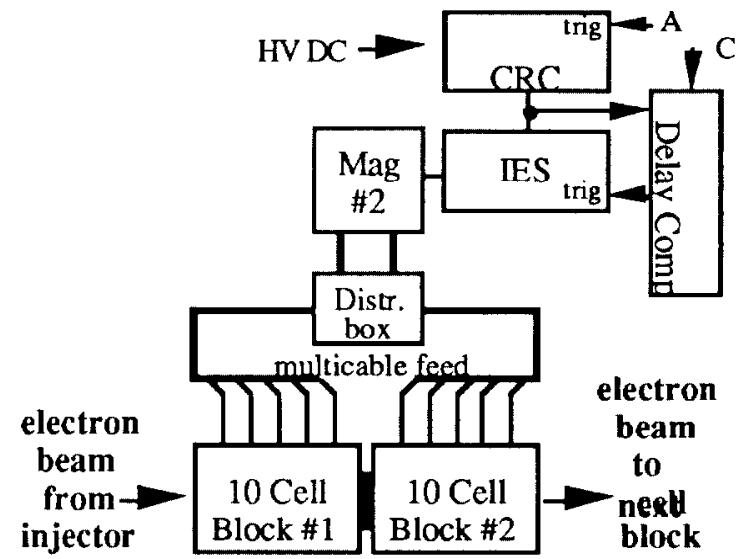

Fig. 1 A typical Pulsed Power system drove 20 cells.

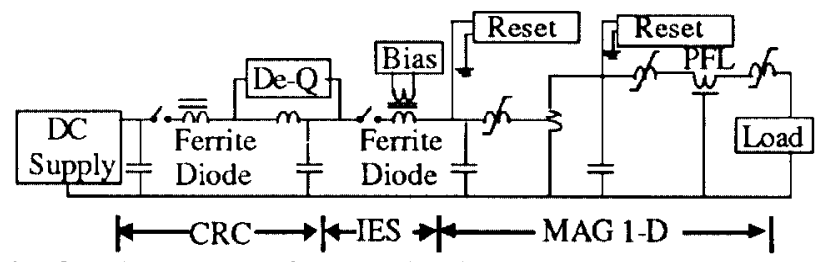

Fig. 2 Pulsed Power Schematic (from Ref. 3).

The first stage of each PPU was the Charge Resonant Command (CRC) circuit, which regulated the voltage. The 
second stage was the Intermediate Energy Storage (IES) which was triggered to fire the accumulated charge into the MAG-1D.

Thyratron tubes were used as switches in the PPU's. The PPU's, the MAG-1D's, and the accelerator cells all contained ferrite material to act as magnetic switches. The ferrites were reset to the bottom of their hysteresis loops by currents in field windings.

The PPU typically generated a voltage pulse of $20-22 \mathrm{kV}$ peak value. This pulse was compressed by the MAG-1D units, which were able to step up the peak voltage to over $130 \mathrm{kV}$. The output pulse had a full width at half maximum of $70 \mathrm{nS}$.

The injector was run by a dedicated PPU and MAG-1D unit, ordinarily at $100-120 \mathrm{kV}$ peak voltage. Three other PPU's each ran a MAG-1D, which each in turn drove 20 accelerator cells in parallel at $80-100 \mathrm{kV}$ peak. A distribution box split the output of each MAG-1D into 10 cables, each of which drove two cells. The cable length provided transit time isolation during the pulse, protecting cells from reflected voltage originating at cells attached to other cables.

Changes made to the pulsed power system for burst mode included an additional high voltage DC power supply and capacitor banks for the PPU's. Thyratron tubes were replaced with high repetition rate units, new ferrite diodes were installed in the PPUs, and new bias circuits were made for the IES reset. MAG-1D pre-compression reset inductors were installed, thyratron trigger chassis were modified, and the DC power supply for the trigger chassis was upgraded. The high voltage trigger distribution units were improved, and circulation pumps were added for the fluid in the cell resistors [4].

\section{B. IMP Wiggler}

The IMP wiggler [6] is a steady-state hybrid laced wiggler with a period of $10 \mathrm{~cm}$ and an active magnetic length of $545 \mathrm{~cm}$. This replaced the pulsed ELF wiggler to maintain resonance during long bursts. The wiggler field in the upstream resonance region, tunable from $2700 \mathrm{G}$ to $5500 \mathrm{G}$, was ordinarily near $3 \mathrm{kG}$. The downstream part had a linear range of $600-4100 \mathrm{G}$. Each electromagnet was cooled by separately instrumented and interlocked water lines. Independently computer-controlled power supplies were used to drive the electromagnets. A copper circular wave guide of $3.25 \mathrm{~cm}$ inner dimension was used between the wiggler magnet poles. The wiggler field errors were measured during assembly at $0.2 \%$ RMS.

The computer control of the wiggler allowed repeatable remote-controlled tapering of the wiggler field without physically moving magnets. Different magnet configurations were stored and re-loaded easily. The computer was also helpful in performing scans of the wiggler ficld, raising and lowering whole sets of magnet poles together.

Because of the permanent magnets, when lower field was selected on any pole, the computer ran an hysteresis loop of the whole wiggler to repeatably arrive at the requested field.

\section{Timing Compensation System}

The feedback timing compensation system for the highvoltage pulses was expanded to handle four MAG-1D pulse compression units independently. The previous singlecorrection LabVIEW ${ }^{\circledR}$ control system [5] was modified by using a LeCroy 2228A Time-to-Digital Converter to measure the firing time of each MAG-1D, from capacitive probe samples of the output. The computer adjusted the delay generator triggering each of the PPU's to cause firing at the target time.

For burst mode, a Kinetics List Sequencing Crate Controller was used to record the delay values for each of the 50 pulses of a burst. Timing corrections were written to a Digital-to-Analog converter. The analog voltages, one for each pulse of a burst, went to a delay compensation chassis for each PPU, which converted the voltage into a trigger delay [7].

The timing system was able to time-align the pulses to within $2-4 \mathrm{nS}$ of the target value when the jitter in the MAGs and PPU's was low (normal operation).

To achieve operation at normal voltage $(80 \mathrm{kV})$ on the accelerator gaps and $2300 \mathrm{Amps}$ injector current (FEL parameters), the accelerator was started at low voltage and raised slowly. This was because the voltage pulses had to be precisely timed with the beam load to avoid over-voltage and gap arcs. Changing voltage caused large timing changes in the PPU's.

For burst mode at full current, it was necessary to start with 2-or 3-pulse bursts at low voltage and slowly raise voltage. The operators found that a sodium thiosulfate solution giving a resistance of $150 \mathrm{Ohms}$ in the cell load resistors prevented arcing and allowed easier startup than de-ionized water, and did not place an unreasonable load on the MAG-1D units. This was due to the resistors providing some load even if beam loading was poor. Ideal beam load was $40 \mathrm{Ohms}$.

\section{EXPERIMENTAL RESULTS}

The injector was operated independenty at up to $3 \mathrm{kHz}$, in bursts of 50 pulses. The entire accelerator was run at $2 \mathrm{kHz}$ because some trigger chassis required reduced inductance in ferrite diodes to run at higher rates This was not done due to time constraints. Repcatablc opcration of up to 50 pulse bursts of electron beam at over $2 \mathrm{kHz}$ and over $6 \mathrm{MeV}$ was achieved. A 45-pulse burst is shown in Fig. 3.

Short bursts of FEL pulses were repeatedly obtained with peak power near $1 \mathrm{GW}$ (Fig 4). For longer bursts, the microwave output was less repeatable but still produced many good pulses (Fig 5).

The reduced effectiveness of long bursts is at least partially attributed to the beam falling out of resonance in the wiggler, due to the pulsed power capacitor banks being drained during the burst. Power supplies connected to the banks were able to recharge capacitors between bursts, but not maintain voltage during a burst. For long bursts, the capacitor bank supplying the injector was drained so that less beam current was produced (Fig. 6). Due to reduced load on the induction gaps, this most likely increased the beam energy unless gap voltage dropped at the corresponding rate. 
In some cases, FEL microwave output power dropped off during a burst due to a mode shift in the gyrotron master oscillator, seen as a change in gyrotron microwave monitors.

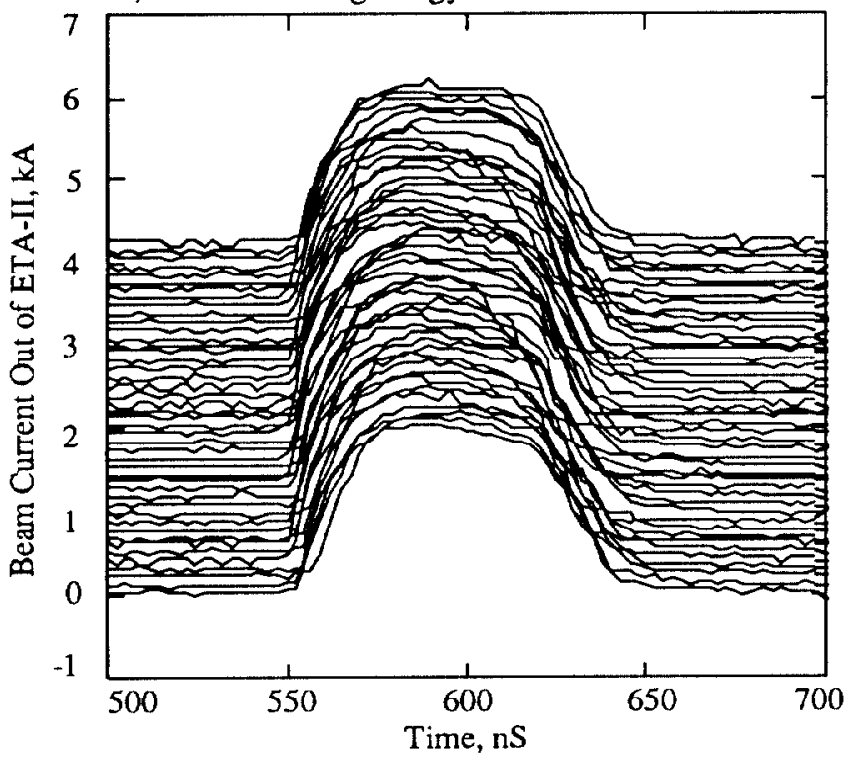

Fig. 3 Electron Beam Current vs. Time for a Burst of 45 Pulses of Electron Beam at $6 \mathrm{MeV}$.

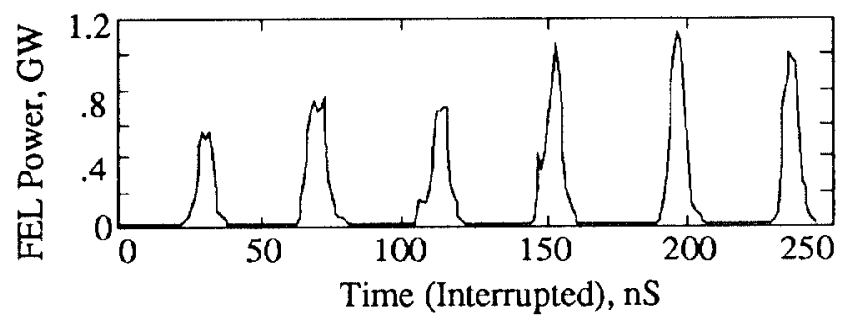

Fig. 4 Microwave Power vs. Time for a Short FEL Burst. The time axis is interrupted: the dead time between pulses recurring at a $2 \mathrm{kHz}$ rate has been removed.

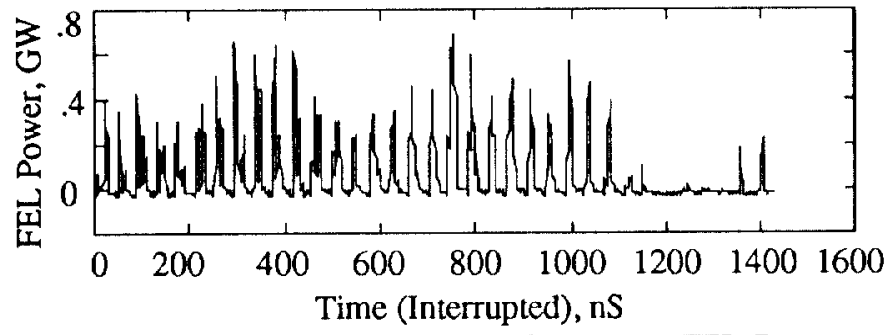

Fig. 5 Microwave Power vs. Time for a Long FEL Burst.

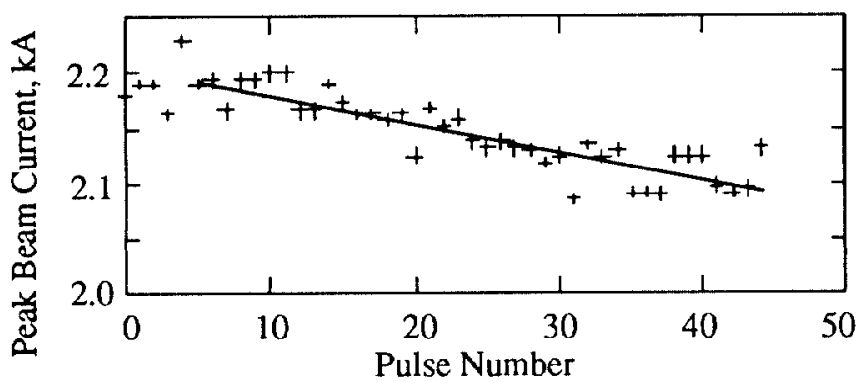

Fig. 6 Peak Current vs. Pulse Number in a 45-Pulse Burst. Peak current falls off slightly during the burst as capacitor banks are drained. The line is a least-squares fit to the points.
The FEL microwave power was measured by a diode which detected a sample of the wiggler output power, by means of a small coupling hole in the center of the first mirror of an evacuated quasi-optical transport system. The diode was calibrated by calorimetry at the end the transport section [8].

\section{DISCUSSION}

Burst Mode Free Electron Laser operation using an induction linear accelerator has been demonstrated for the first time, and was used to produce bursts of high power $(\sim 1 \mathrm{GW})$ pulsed microwaves. The accelerator hardware was upgraded for high repetition rate, and the timing compensation system was expanded to control burst mode timing synchronization.

There is potential for improvement. Using a much larger power supply ( $5 \mathrm{MW}$ compared to $695 \mathrm{~kW}$ total for ETA-III), the High Average Power Test Stand at LLNL was able to consistently demonstrate operation of a PPU and MAG-1D pulse compressor into a dummy load for 800 -pulse bursts (a result of the work described in ref [4]). Burst length could also be extended by using larger capacitor banks.

\section{REFERENCES}

[1] S.L. Allen et al., "Generation of High Power $140 \mathrm{GHz}$ Microwaves with an FEL for the MTX Experiment," these proceedings

[2] S.L. Allen et al. "Measurement of Reduced Corkscrew Motion on the ETA-II Linear Induction Accelerator", Proceedings of the 1991 Part. Accel. Conf. (San Francisco, 1991) 3094

[3] W.C. Turner, "Control of Energy Sweep and Transverse Beam Motion in Induction Linacs," Proc. 1991 Part. Accel. Conf. (San Francisco, 1991) 3097.

[4] S.E. Sampayan, et al., "Performance Characteristics of an Induction Linac Magnetic Pulse Compression Modulator at Multi-Kilohertz Pulse Repetition Frequency," Proc. 1991 Part. Accel. Conf. (San Francisco, 1991) 3097

[5] Previous Single-pulse timing system by F.E. Coffield

[6] B. Felker and S.W. Ferguson, "The Intense Microwave Prototype (IMP) Free Electron Laser, 140 Gigahertz Microwave System for the Microwave Tokamak Experiment (MTX)", Proc. 14th IEEE Symposium on Fusion Engineering (San Diego, 1991)

[7] M.A. Newton and J.A. Watson, "Timing and Voltage Control for Magnetic Modulators on ETA-II," Proc. 7th IEEE Pulsed Power Conf. (Monterey, CA 1989)

[8] B.W. Stallard et al., "ECH by FEL and Gyrotron Sources on the MTX Tokamak," in Proceedings of the 16th Symp. on Fus. Tech., London, Sept. 1990. 